\title{
New Methods for Solving Integral - Differential Transport Equations
}

\section{Olga Martin*}

Polytechnic University of Bucharest, Faculty of Applied Sciences, Splaiul Independentei 313, Bucharest, Romania

The integral-differential equations describe many phenomena in different field of mechanical and nuclear engineering, chemistry, astronomy, biology and potential theory. The resolution of boundary problems for these equations are the subject of several papers in which the authors have approached in most cases numerical methods: Monte Carlo, truncated series of Chebyshev polynomials, the fictitious domain method, $\mathrm{S}_{\mathrm{N}}$ method and the finite element method [1-4]. An exact solution was found only in the particular cases. Generally, these are obtained with the help of the methods of mathematical analysis, abstract functional analysis and the spectral methods [5].

In recent years, the basic ideas of the homotopy, which is a concept of the topology and differential geometry, were used to obtain the approximate solutions for a wide class of differential, integral and integral-differential equations. We mention here the homotopy perturbation method (HPM) proposed by Ji-Huan He in 1998 and the homotopy analysis method (HAM) proposed by Liao in 1992. The perturbation methods approximate the solution of given problem by a series of small parameters. Unfortunately, the majority of non-linear problems have no small parameters and an unsuitable choice of these parameters can lead to bad effects. The new homotopy perturbation technique (HPM) embeds a parameter $p$ that ranges from zero to one $[6,7]$. When the embedding parameter is zero, we get a linear equation and if it is equal to one, we get the original transport equation. This embedding parameter that belongs to the interval $(0,1)$ can be considered as a small parameter.

The homotopy analysis method (HAM) is one of the most effective methods to obtain the exact and approximate series solutions for the integral-differential equations [8]. Being a great freedom in choosing of the initial guess functions and the auxiliary functions, are proposed for these such forms that correspond to the conditions imposed at the ends of the spatial interval and thus the algorithm becomes fast convergent. In the papers [9-11] is shown that the homotopy perturbation method, variational iteration method and Adomian decomposition method are only special cases of the homotopy analysis method solutions. Today, a new homotopy method is added to the above. With this we can solve the multipoint boundary problem for a stationary transport equation in the non-homogeneous media. Comparative studies between the solutions obtained with the help of mentioned algorithms for a numerical example with the appropriate exact solutions illustrate the accuracy and computational efficiency of these new methods.

\section{References}

1. Halton JH (1994) Sequential monte carlo techniques for the solution of linear systems. J Sci Comput 9: 213-257.

2. Lewis E, Miller WF (1993) Computational methods of neutron transport American Nuclear Society, Illinois, USA.

3. Martin $O$ (2009) Approximate method for solving a neutron transport equation. $\mathrm{J}$ Computational Analysis and Applications 11: 431-442.

4. Martin $O$ (2009) On approximation in the fictitious domain method for a bidimensional transport equation. Num Meth Partial Diff Eqs 26: 1275-1290.

5. Martin $\mathrm{O}$ (2007) Variational formulation of a mixed problem for an integral differential equation. J Computational Analysis and Applications 9: 311-318.

6. Bizar J, Ghazvini H (2008) Numerical solution for special non-linear Fredholm integral equation by HPM. Appl Math Comput 198: 681-687.

7. Martin $O$ (2011) A new homotopy perturbation method for solving a neutron transport equation. Applied Mathematics and Computation 217: 8567-8574.

8. Matinfar M, Saeidy M, Vahidi J (2010) Analytical solution of BVPs for fourth-order integro-differential equations by using HAM. Int J Nonlinear Sci 9: 414-421.

9. He JH (2004) Comparison of homotopy perturbation method and homotopy analysis method. Appl Math Comput 156: 527-539.

10. Li TY, Wang X (1993) Solving real polynomial system with real homotopies. Math Comput 60: 669-680.

11. Liao S (2009) Notes on the homotopy analysis method: some definitions and theorems. Commun Nonlinear Sci Numer Simul 14: 983-997.
${ }^{*}$ Corresponding author: Olga Martin, Professor, Polytechnic University of Bucharest, Faculty of Applied Sciences, Splaiul Independentei 313, Bucharest, Romania,E-mail: omartin_ro@yahoo.com

Received July 26, 2012; Accepted July 29, 2012; Published August 03, 2012

Citation: Martin O (2012) New Methods for Solving Integral-Differential Transport Equations. J Applied Computat Mathemat 1:e116. doi:10.4172/21689679.1000e116

Copyright: (c) 2012 Martin O. This is an open-access article distributed under the terms of the Creative Commons Attribution License, which permits unrestricted use, distribution, and reproduction in any medium, provided the original author and source are credited. 